\title{
A Review of Management of Inflammation in the HIV Population
}

\author{
Jihad Slim ${ }^{1,2,3}$ and Christopher F. Saling ${ }^{3,4}$ \\ ${ }^{1}$ Saint Michael's Medical Center, Newark, NJ 07102-2094, USA \\ ${ }^{2}$ Saint Joseph's Regional Medical Center, Paterson, NJ 07503, USA \\ ${ }^{3}$ New York Medical College, Valhalla, NY 10595, USA \\ ${ }^{4}$ Internal Medicine, Saint Michael's Medical Center, Newark, NJ 07102, USA \\ Correspondence should be addressed to Christopher F. Saling; christopher.saling@gmail.com
}

Received 26 May 2016; Revised 12 August 2016; Accepted 25 August 2016

Academic Editor: Barbara Rossetti

Copyright (C) 2016 J. Slim and C. F. Saling. This is an open access article distributed under the Creative Commons Attribution License, which permits unrestricted use, distribution, and reproduction in any medium, provided the original work is properly cited.

\begin{abstract}
Advancements in antiretroviral therapy have drastically increased the life expectancy for those infected with HIV. Today, a new subgroup of older patients with long-term controlled HIV exists, and its populace is continuously mounting. Therefore, it is essential to understand the enduring effects of chronic suppressed HIV infection in order to further improve HIV management in these patients. This paper will examine the role of HIV in chronic inflammation and immune dysfunction, the dynamic interaction that exists between comorbidity and HIV, and the potential consequences of long-term antiretroviral therapy in an effort to provide the best management options for the virally suppressed HIV patient.
\end{abstract}

\section{Introduction}

Before the advent of effective cART, HIV infection was usually a death sentence. The virus would hijack the immune system unchecked, often leading to full-blown AIDS and acute opportunistic infection. According to the CDC, there were a total of 774,467 diagnosed cases of AIDS and 448,060 AIDS-related deaths in the USA from 1981 to 2000 [1]. Over the past two decades, cART has dramatically reduced the incidence of morbidity and mortality related to both HIV and opportunistic infections. These advancements have enabled most persons living with HIV (PLWH) to achieve an undetectable viral load (VL) within 12 weeks of the initiation of treatment. This has led to the emergence of a new and growing population of aging HIV-positive patients. The effects of chronic suppressed HIV infection in this group are only beginning to become understood, and methods to combat these effects are poorly studied.

Although the average life expectancy for PLWH has increased significantly, it is still slightly less than that of the general population. The highest estimated life expectancy for a newly diagnosed HIV-positive 20 -year-old patient on cART in the USA or Canada is just above 70 years [2]. Reasons for this gap have become a new focus of HIV research. One theory is that even with undetectable $\mathrm{VL}$ and adequate $\mathrm{CD} 4^{+}$ count there exists a state of persistent inflammation. Also, traditional comorbidities may become worsened by chronic HIV infection. Furthermore, long-term use of cART may lead to adverse effects that could further augment the severity of these comorbidities. This paper will focus on each one of these concepts in order to provide possible treatment measures to thwart their harmful effects on patients with suppressed HIV infection.

\section{Materials and Methods}

A computer-based search using Pubmed and Embase was conducted to review the literature regarding antiretroviral interventions to decrease chronic inflammation in patients on combination antiretroviral therapy (cART).

\section{Results and Discussion}

3.1. Inflammation and Dysregulation of the Immune System. Inflammatory markers appear to directly correlate with morbidity and mortality in patients infected with HIV. Since 
Immune dysregulation relationship to comorbidities in patients with HIV

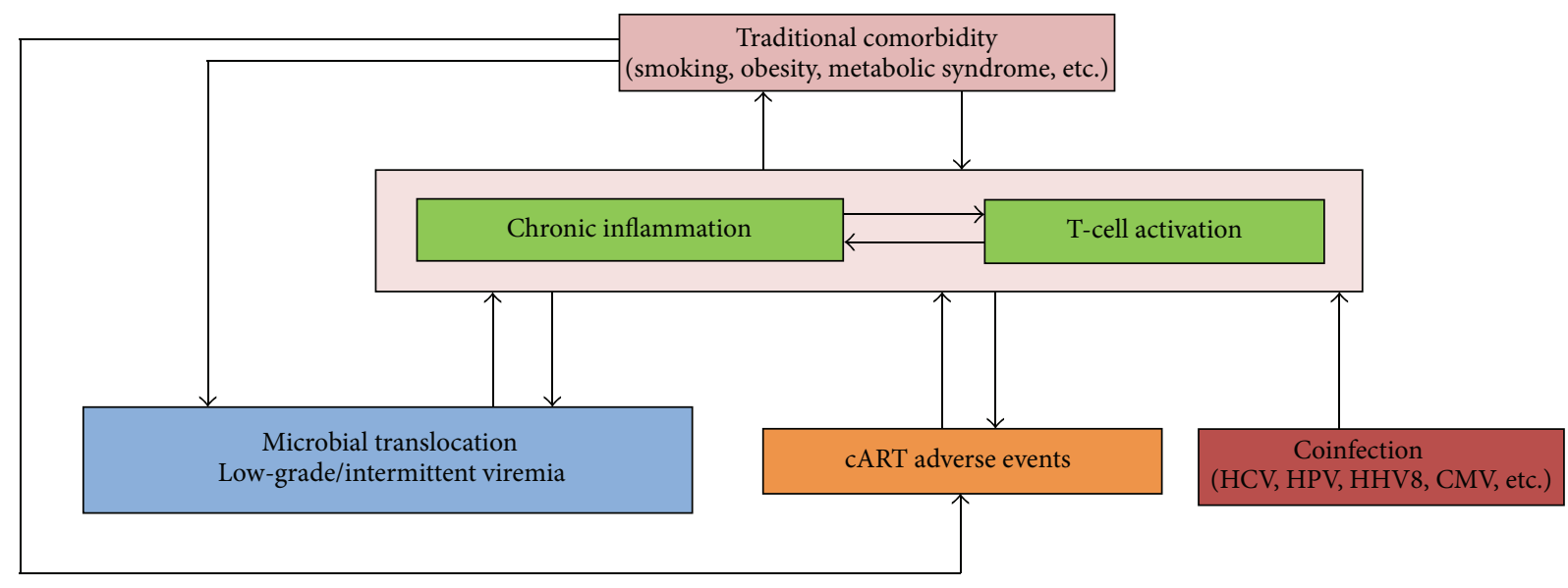

FIGURE 1: Microbial translocation, low-grade viremia, coinfection, and adverse events from cART all contribute towards chronic inflammation and T-cell activation. This immune dysfunction augments the severity of traditional comorbid conditions. Furthermore, the comorbidities themselves intensify all of the aforementioned factors causing a positive feedback cycle.

this inflammatory process, which is a correlate of T-cell activation, is much more pronounced when the virus is not suppressed, the discussion will be limited towards patients receiving effective treatment for HIV [3]. Although successful cART does not suppress all inflammatory mechanisms associated with HIV, it has been shown to decrease some immune activation markers to the level of HIV-uninfected individuals, particularly monocyte-macrophage activation [4]. However, in clinical practice HIV virus load (VL) is measured intermittently and there are different cut-offs for detection. Thus, it is conceivable that low-grade or intermittent viremia is actually occurring in patients that are classified as undetectable. This phenomenon could play a role in persistent inflammation.

It is also relatively well established that a chronic inflammatory state in patients receiving appropriate cART is primarily related to the extent of damaged gut-associated lymphoid tissue and its subsequent microbial translocation $[5,6]$. This process involves commensal microbes from the gastrointestinal tract entering portal and systemic circulation [7]. Acute HIV infection is marked by an intense surge of cytokines such as interferon- $\alpha$, interferon- $\gamma$, tumor necrosis factor, and IL- 6 which leads to immune activation and severe inflammatory reaction [8]. This causes a profound depletion of $\mathrm{CD}^{+}$cells from the gut, which only partially improves with effective cART $[9,10]$. This explains the reasoning behind intestinal microbial translocation and subsequent immune stimulation [11]. One method for studying microbial translocation is through the measurement of serum lipopolysaccharide (LPS). The SMART study revealed that soluble CD14, a marker of monocyte response to LPS, was an independent predictor of mortality in PLWH [12]. This was corroborated by another case-control study by Hunt et al., which concluded that gut epithelial barrier dysfunction independently predicts mortality in individuals with treated HIV infection who also have a history of AIDS [13].

Another factor relating to inflammation in PLWH is Tcell function. It is unclear if immune dysregulation leads to inflammation or vice versa. Nonetheless, they are usually present together and both contribute to the burden of comorbid illnesses [14]. Hunt et al. studied the relationship of immune activation and increased CD4 count when HIV was suppressed with cART and found that increased Tcell activation was associated with shorter duration of viral suppression, HCV coinfection, frequent low-level viremia, lower nadir $\mathrm{CD}^{+}$T-cell counts, and a lower gain in $\mathrm{CD} 4^{+}$ T-cells [15]. In an elegant study of impaired gut junctional complexes by Tincati et al., a relationship was established between gut damage, HIV viral reservoir, and $\mathrm{CD} 4^{+}$response to cART [5]. They concluded that the more damage to the gut and the larger the reservoir, the less the increase in $\mathrm{CD} 4^{+}$cells while on suppressive cART [5]. Figure 1 illustrates the factors involved in the chronic inflammatory state and immune dysfunction in PLWH with controlled viral load.

It is also postulated that coinfection is a key contributor to immune dysregulation present in PLWH receiving suppressive cART. This association was well established by Masiá et al., who prospectively studied multiple blood biomarkers of inflammation in monoinfected HIV patients compared to those coinfected with HHV8 [16]. Both groups had a suppressed HIV VL, but inflammation and immune activation were significantly higher in those with HHV8 coinfection [16]. Since HCV is another prevalent virus found in $20-25 \%$ of PLWH, it is relevant to study its impact on immune recovery as well. Zaegel-Faucher et al. retrospectively reviewed this data in patients with undetectable VL for at least 3 years and concluded that $\mathrm{CD} 4^{+}$percentage and CD4/CD8 ratio were lower in patients coinfected with $\mathrm{HCV}$ compared to those with monoinfection, even though they had similar cART regimen and $\mathrm{CD}^{+}$and $\mathrm{CD}^{+}$counts at first undetectable HIV VL [6]. Furthermore, Modjarrad and Vermund reviewed the literature up to April 2010 and found that treatment of Mycobacterium tuberculosis, syphilis, and other infections significantly decreased HIV VL, even when no cART was used [17]. In another prospective study using 
CMV PCR, Deayton et al. established a direct correlation of positive PCR findings with new AIDS-defining disorders and mortality in PLWH in the highly active cART era [18]. Lastly, in their review of CMV in PLWH, Barrett et al. summarized the evidence that CMV could be an important cofactor in the development of age-related morbidities in HIV infection [19].

The pivotal SMART trial provided strong evidence of the association of inflammatory biomarkers and coagulation with increased risk of all-cause mortality [20]. The study also showed that interleukin-6 (IL-6) and D-dimer were significantly associated with increased risk of CVD and other causes of death, even in patients on cART [21]. Tenorio et al. conducted a case-control study that concurred with these findings, concluding that soluble inflammatory markers correlated with non-AIDS-defining events in patients virally suppressed on therapy [22].

3.2. The Role of Traditional Comorbidity and Coinfection. The new challenges facing the HIV-infected population are nonAIDS-related conditions. A prospective HIV cohort study by the $\mathrm{D}: \mathrm{A}: \mathrm{D}$ group found that the most common comorbidities leading to death in HIV patients were non-AIDS cancers, cardiovascular disease (CVD), and liver disease [23]. Morlat et al. expressed these same findings in a study in France in 2010 [24]. A Swiss HIV cohort study by Hasse et al. revealed that stroke, myocardial infarction, diabetes mellitus (DM), fragility bone fractures, and non-AIDS-defining malignancies were significantly elevated for persons aged $\geq 65$ years [25]. Guaraldi et al. performed a case-control study on cARTexperienced patients treated at Modena University, Italy, from 2002 to 2009 [26]. They were compared with age-, sex-, and race-matched adults from the general population [26]. They specifically looked at noninfectious comorbidities (NICMs), which included CVD, hypertension, diabetes mellitus (DM), bone fractures, and renal failure [26]. The study defined polypathology (Pp) as the concurrent presence of $\geq 2$ NICMs and concluded that the prevalence of Pp among HIV patients aged 41-50 years was similar to that among controls aged 51-60 years [26]. Logistic regression models showed that independent predictors of $\mathrm{Pp}$ in the overall cohort were age (odds ratio OR, 1.11), male sex $(\mathrm{OR}, 1.77)$, nadir $\mathrm{CD} 4^{+}$count $<$ 200 cells $/ \mu \mathrm{L}$ (OR, 4.46), and cART exposure (OR, 1.01) [26].

Multiple studies have identified HIV as an independent risk factor for acute myocardial infarction (AMI). Freiberg et al. reviewed data from participants in the Veterans Aging Cohort Study that included both HIV-infected and noninfected individuals [27]. This study concluded that infection with HIV was associated with a 50\% increased risk of AMI beyond which was explained by recognized risk factors [27]. In two cohorts from the Partners HealthCare System in Boston, Triant et al. compared the rate of AMI in HIVpositive and HIV-negative patients while adjusting for age, gender, race, hypertension, DM, and dyslipidemia [28]. They, too, concluded that there was an increased risk of AMI in patients with HIV, especially in HIV-positive women [28]. Lastly, Okeke et al. reviewed the hospital discharge data from the Nationwide Inpatient Sample from 2002 to 2012 looking specifically for patients with AMI or stroke [29]. They used multivariable logistic regression to evaluate the association between HIV and in-hospital death [29]. They found that patients with a history of AIDS were significantly more likely to die in-hospital after AMI and stroke than noninfected patients [29]. This disparity was not observed when infected patients without a history of AIDS were compared to noninfected patients [29].

Malignancies that are considered AIDS-related such as Kaposi's sarcoma, primary central nervous system lymphoma, and cervical cancer have dramatically declined since the advent of suppressive ART [30]. Furthermore, the incidence of non-AIDS-related malignancies (NARM) including anal cancer, hepatocellular carcinoma, head and neck cancers, lung cancers, non-Hodgkin's lymphoma, and melanoma has increased so significantly that they now represent one of the most common causes of death in PLWH in the USA [31]. Immune dysregulation and chronic inflammation in PLWH can promote increased cell proliferation and generate potentially damaging reactive oxygen species [32]. The immune dysfunction associated with HIV infection may also lead to impaired immune surveillance with an impaired ability to both detect and eliminate early tumor cells [33]. Powles et al. reported from their large prospective cohort that a nadir $\mathrm{CD} 4^{+}$count $<200 /$ microL had a significant association with NARM [34]. Certain traditional risk factors for NARM are more prevalent in PLWH $[35,36]$. Some of these include smoking, with its subsequent increase in lung cancer, as well as hepatitis $B$ and $C$ viruses, with their associated risk for hepatocellular carcinoma $[35,36]$. There is a higher incidence of Hodgkin's lymphoma in PLWH than the general population, and it is often associated with EBV coinfection [37]. Probably the highest increase in cancer types in HIVinfected patients compared to those that are noninfected is related to HPV [38]. Associated cancer types with this coinfection include anal cancer, head and neck cancer, and cervical cancer (which is an AIDS-related malignancy) [38].

The incidence of DM is also increased in PLWH [39]. Inflammation and HIV lipodystrophy involve adipose tissue redistribution, mitochondrial dysfunction, altered differentiation of adipocytes, and increased adipocyte lipolysis [40, 41]. This leads to altered adipokine secretion, as well as the release of proinflammatory cytokines and free fatty acids $[40,41]$. This, in turn, exacerbates chronic inflammation, dyslipidemia, and insulin resistance [40, 41].

The effect of HIV on liver disease was well characterized by Towner et al. in their case-control study which concluded that HIV-infected individuals have a higher risk of hepatic dysfunction and hepatic-related death compared to those without HIV infection, even with adjustment for known hepatic risk factors [42]. Marchetti et al. revealed that HIV coinfected patients, mainly HCV, with higher TNF- $\alpha$ plasma levels had a 13-fold increase in the risk of progression to a Fib-4 > 1.45 [43]. However, these patients were not receiving ART [43]. A more recent study suggests that ART with good immune reconstitution can slow down liver fibrosis in HIV/HCV coinfected patients [44].

3.3. Chronic Effects of cART. With the ever-emerging population of patients with long-term controlled HIV infection, the chronic effects of cART are becoming better understood. 
It has already been well established that a high proportion of patients who delay cART until the $\mathrm{CD} 4^{+}$count drops below 200 cells $/ \mathrm{mm}^{3}$ do not achieve a normal $\mathrm{CD} 4^{+}$count, even after a decade of effective therapy [45]. In fact, the DHHS guidelines now recommend usage of cART in all patients regardless of $\mathrm{CD} 4^{+}$count [46]. Furthermore, studies have suggested that $\mathrm{CD}^{+}$cell recovery from cART is both slower and less pronounced in elderly patients, which provides further credence to the concept that early cART initiation will lead to the best possible degree of immune regulation [47, 48]. These guidelines are supported by multiple randomized controlled studies like START and TEMPRANO proving that the earlier ART is started the better the immune system is preserved and the less inflammation is present, with subsequently less mortality and morbidity $[49,50]$.

cART must also be considered a potential cause of adverse events and, therefore, a possible contributor towards inflammation and aging in those with controlled HIV infection [51]. Extra attention to drug toxicity from cART must be given to the elderly HIV patient due to the high degree of concurrent medication use and, thus, the greater potential for harmful drug-drug interactions, as well as age-related changes in renal and hepatic function that could affect drug clearance.

Certain regimens have been linked to toxicities that increase the risk for comorbidities. This paper has already mentioned the role of comorbidity in persistent inflammation. cART can further negatively contribute towards these processes by augmenting the severity and effects of comorbid conditions. Friis-Møller et al. studied the association between cART and CVD risk factors and found that nonnucleoside reverse transcriptase inhibitor (NNRTI) and protease inhibitor (PI) use was linked to elevated total cholesterol [52]. The D:A:D Study Group added further to this subject by investigating the association between certain cART drugs and acute myocardial infarction (AMI) [53]. The study revealed that abacavir, didanosine, indinavir, and lopinavir-ritonavir carried the most significant association [53]. Lang et al. further contributed to this subject, stating that cumulative exposure to PIs, especially (fos)amprenavir with or without ritonavir and lopinavir with ritonavir, had increased risk for AMI [54]. However, saquinavir was found not to have this association [54]. Lopinavir and ritonavir were also shown to increase the risk for AMI in a study conducted by Durand et al. [55].

Abacavir increasing the risk for AMI has been a topic of debate. A paper by Marcus et al. addressed this issue and concluded that abacavir increases the risk of CVD by 2.2 times [56]. Two other studies, one by the D:A:D group and another by Obel et al., also confirmed that abacavir is associated with AMI $[57,58]$. In their cohort study, Choi et al. concluded that recent abacavir exposure increased the risk for cardiovascular events and that tenofovir (TDF) was associated with heart failure [59]. Costagliola et al., however, reviewed the literature on abacavir and CVD and reported that due to confounding variables and selection bias, it is impossible to neither verify nor repudiate a correlation between the two [60]. Many studies claim that abacavir use carries no independent risk for AMI [61-64]. Bedimo et al. found no association between
MI use and abacavir use but did report that abacavir use was more common than TDF use in patients with chronic kidney disease, which itself is an independent risk factor for CVD [62]. Nonetheless, the DHHS guidelines suggest avoiding abacavir and lopinavir/ritonavir in patients at high risk for cardiovascular events [46].

Other associations between certain cART toxicities and comorbidities have been established in the literature. Ryom et al. found that cumulative use of stavudine, didanosine, (fos)amprenavir, and TDF was independently associated with higher rates of end stage liver disease and hepatocellular carcinoma [65]. In their prospective observational study, De Wit et al. showed that stavudine and zidovudine were linked with insulin resistance, perhaps due to toxic effects on the mitochondria [66]. Moreover, TDF and efavirenz have been linked with decreased bone marrow density [67]. In their meta-analysis, Brown and Qaqish concluded that PItreated patients had increased odds of osteoporosis than non-PI-treated patients [68]. Also, a study by Perrot et al. linked TDF use to osteomalacia from proximal renal tubular dysfunction [69]. Herlitz et al. further studied the potential adverse events of TDF use by documenting reversible acute tubular necrosis and mitochondrial dysmorphic changes in 13 cases of TDF-associated nephrotoxicity that were on therapy for a mean of 19.6 months [70]. Two controlled, double-blind phase 3 studies by Sax et al. compared the adverse events associated with TDF with those of its newer formulation tenofovir alafenamide (TAF) [71]. They concluded that TAF has less renal and bone adverse events than TDF because this tenofovir prodrug causes a $90 \%$ decrease in tenofovir plasma concentration [71]. Also, TAF was noninferior to TDF in suppressing HIV VL [71]. Additionally, Leeansyah et al. studied telomerase activity and length in vitro by looking at peripheral blood mononuclear cells (PBMCs) from HIV-infected patients receiving a NRTI-containing regimen and found that they had significantly lower telomerase activity than both HIV-uninfected patients and HIV-infected patients receiving a non-NRTI-containing regimen [72]. Telomerase length was inversely associated with age, as well as the total duration of NRTI-containing therapy [72]. This study concluded that NRTIs at therapeutic concentrations, specifically TDF, inhibit telomerase activity and this leads to its accelerated shortening in activated PBMCs, which could play a role in the enhanced aging of PLWH [72]. This is important because some studies have reported that the HIV virus itself causes shortening of leukocyte telomere length, and, therefore, TDF use may further contribute towards this same process $[73,74]$.

3.4. Managing Inflammation in the HIV Patient. The management of cART in the HIV-infected patient must focus on the need not only to suppress the serum HIV VL but also to target potential viral reservoirs in order to decrease immune activation and the chronic inflammatory response. One of these possible reservoir sites is within the central nervous system (CNS). In their CROI abstract, Anderson et al. examined the effect of cART therapy with good CNS penetration in virally suppressed patients by documenting inflammatory markers in cerebrospinal fluid (CSF) [75]. They found that, during suppressive cART, regimens that are estimated to 
have better distribution into the CNS were associated with decreased levels of CXCL10 and TNF- $\alpha$ within the CSF and, therefore, less inflammation [75]. IL-6 remained elevated even in regimens with good CNS penetration [75].

Another potential site for these reservoirs is within the gut mucosa [76-78]. Gandhi et al. studied the effects of adding another antiretroviral agent to an already suppressive regimen [79]. They measured any changes in the level of residual viremia in patients with $\mathrm{VL}<20$ copies/mL after adding raltegravir (RAL) for 12 weeks [79]. They found that intensification with RAL did not reduce the amount of residual viremia [79]. Although a study by Yukl et al. echoed the same results for a RAL-containing intensification on the VL in plasma, it did reveal a decrease in T-cell activation within the gut [77]. Perhaps this is because RAL concentrations remain significantly higher in the gut-associated lymphoid tissue (GALT) and gastrointestinal tract than in the plasma, causing less viral replication at these sites [78]. Hatano et al. concurred with the observation that RAL intensification decreases low-level viral replication, as evidenced by their measurement of an increase in the level of 2-long terminal repeat (2-LTR) circles in these patients [80]. Although this is a promising treatment option, other accounts claim that intensification with RAL did not increase overall $\mathrm{CD} 4^{+}$count nor lower HIV proviral DNA in gut $\mathrm{CD} 4^{+}$cells [76].

Other studies have investigated changes in inflammatory markers after switching suppressive cART to RAL [81-84]. The SPIRAL trial took controlled patients on a boosted protease inhibitor (PI) and then randomly switched them in a 1:1 fashion to RAL [82]. Results at 48 weeks after the randomized switch revealed significant changes in several cardiovascular biomarkers that could not be completely explained by lipid changes [82]. Lake et al. reported a decrease in sCD14 in obese women who were changed to RAL who previously had controlled VL on a PI or NNRTI [84]. A study by Silva et al. found a similar decrease in inflammatory markers in virologically suppressed patients that were switched to RAL from an enfuvirtide-based cART [83]. Lastly, Gupta et al. changed patients virally suppressed on efavirenz to RAL and documented that the switch group had decreased C-reactive protein, sCD14, and renal function and increased levels of sCD163 [85]. This suggests that, compared to the continuation group, RAL may more positively impact monocyte activation, but it is not superior in its effects on endothelial function. Furthermore, RAL could contribute more towards nephrotoxicity than efavirenz [85].

Intensification with maraviroc (MVC) has also been tested in virally suppressed patients to determine its effects on $\mathrm{CD}^{+}$cell restoration and immune regulation. Cuzin et al. added MVC for 24 weeks to the cART of 60 patients with $\mathrm{CD}^{+}<350$ cells $/ \mathrm{mm}$ [86]. They found that these patients were able to achieve an increase in $\mathrm{CD} 4^{+}$slopes [86]. Hunt et al. also studied MVC intensification in patients with $\mathrm{VL}<$ 48 copies $/ \mathrm{mL}$ and $\mathrm{CD}^{+}<350$ cells/mm [87]. Compared with the placebo group, MVC-treated subjects had increased peripheral blood $\mathrm{CD}^{+}$cells, sCD14, and neutrophils, with a lesser effect in suppressing $\mathrm{CD} 4^{+}$cell activation by week 24 [87]. Lastly, Belaunzarán-Zamudio et al. compared the immune recovery in patients with $\mathrm{CD}^{+}<100$ cells $/ \mathrm{mm}$ who received either MVC intensification or placebo to a standard cART regimen [88]. Those on MVC retained higher rates of $\mathrm{CCR}^{+}, \mathrm{CD}^{+}$, and $\mathrm{CD}^{+}$cells and had no effect on IRIS occurrence [88].

There have been a small variety of head-to-head trials in treatment naïve patients in order to assess which regimens are most efficacious in diminishing immune activation. Hileman et al. examined markers of inflammation and monocyte activation in a randomized controlled blinded study of single tablet regimen of cobicistat/elvitegravir/emtricitabine/TDF versus efavirenz/emtricitabine/TDF [89]. They concluded that the elvitegravir-containing regimen had a greater decrease in sCD14, hsCRP, and Lp-PLA2 levels compared to the efavirenz-containing regimen [89]. The effects on the inflammatory response by MVC versus TDF were examined in an abstract submitted by Chan et al. [90]. They found that initiating cART with MVC caused a greater increase in $\mathrm{CD} 4^{+}$ cells, a smaller decrease in $\mathrm{CD}^{+}$cells, and a smaller increase in $\mathrm{CD}^{+} / \mathrm{CD}^{+}$ratio compared to TDF [90]. However, there was no difference in changes in inflammatory biomarkers between the two regimens [90]. Papakonstantinou et al. compared the inflammatory effects on treatment naïve patients assigned a regimen of either TDF/emtricitabine/efavirenz or abacavir/lamivudine/efavirenz [91]. The results of the study revealed that the TDF-containing regimen caused a decrease in platelet-activating factor (PAF) levels and lipoproteinassociated phospholipase A2 (Lp-PLA2) activity [91]. The abacavir-containing regimen showed no change in baseline PAF and an increase in Lp-PLA2, which may be the reasoning behind abacavir-associated cardiovascular adverse events [91]. Lastly, Barrios et al. studied TDF + didanosine in patients who were naive to treatment or who simplified a prior suppressive cART regimen [92]. They established that although VL was undetectable, a decline in $\mathrm{CD} 4^{+}$count was evident, which they theorized could be due to an imbalance in adenosine metabolites within $\mathrm{CD} 4^{+}$cells [92]. A summary of the studies regarding cART intervention to manage the chronic inflammatory state in HIV patients can be found in Table 1.

There have also been promising studies suggesting alternate therapies to treat chronic inflammation. Multiple published trials revealed that rosuvastatin showed benefit in reducing inflammation markers and immune activation [9395]. Wooten at al. examined the effect of healthy diet and exercise on inflammation in HIV patients with undetectable VL and dyslipidemia [96]. They found that these interventions effectively reduced plasma Lp-PLA2 mass [96]. Furthermore, patients should be counseled to stop smoking. Valiathan et al. compared HIV-infected smokers and nonsmokers that had documented viral suppression on cART to HIV-uninfected smokers and nonsmokers [97]. They found that smoking and HIV infection both independently influence T-cell immune activation and function, and together they present the worst immune profile [97]. Villar-García et al. conducted a doubleblind, randomized, placebo-controlled trial of Saccharomyces boulardii in 44 patients with viral load of $<20$ copies per milliliter for at least 2 years. They found that this fungus was very effective at decreasing microbial translocation and inflammation parameters [98]. Another innovative approach 


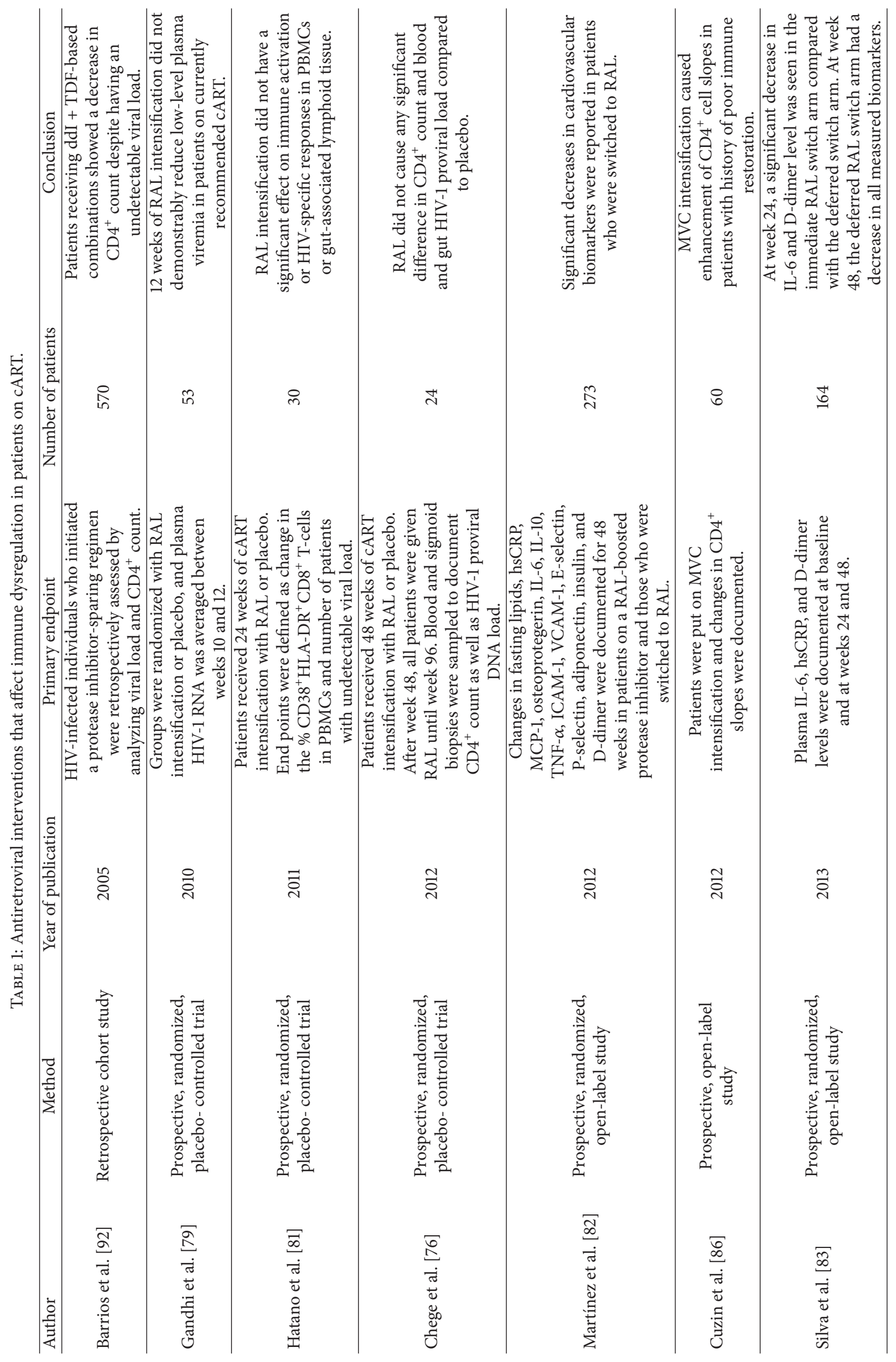




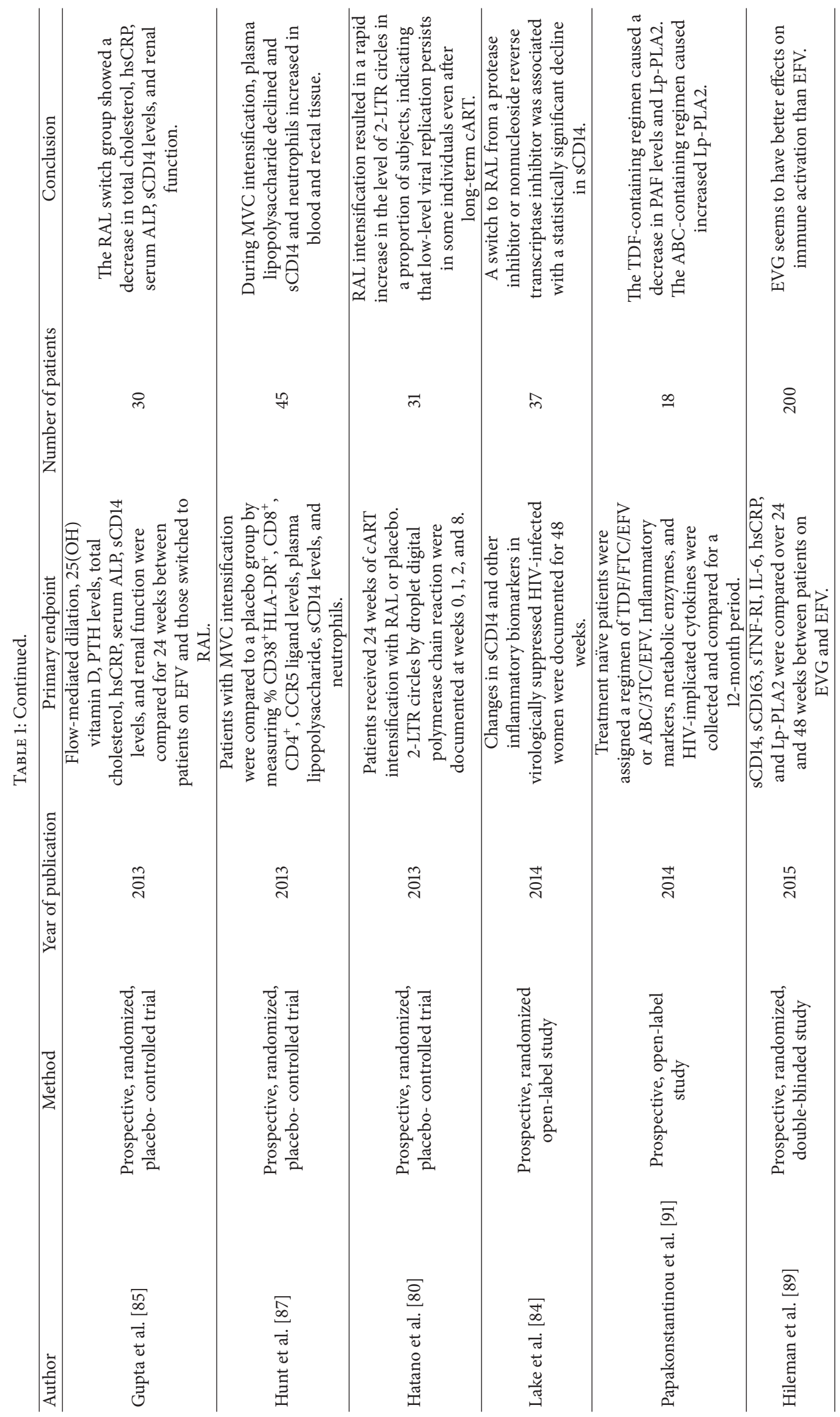




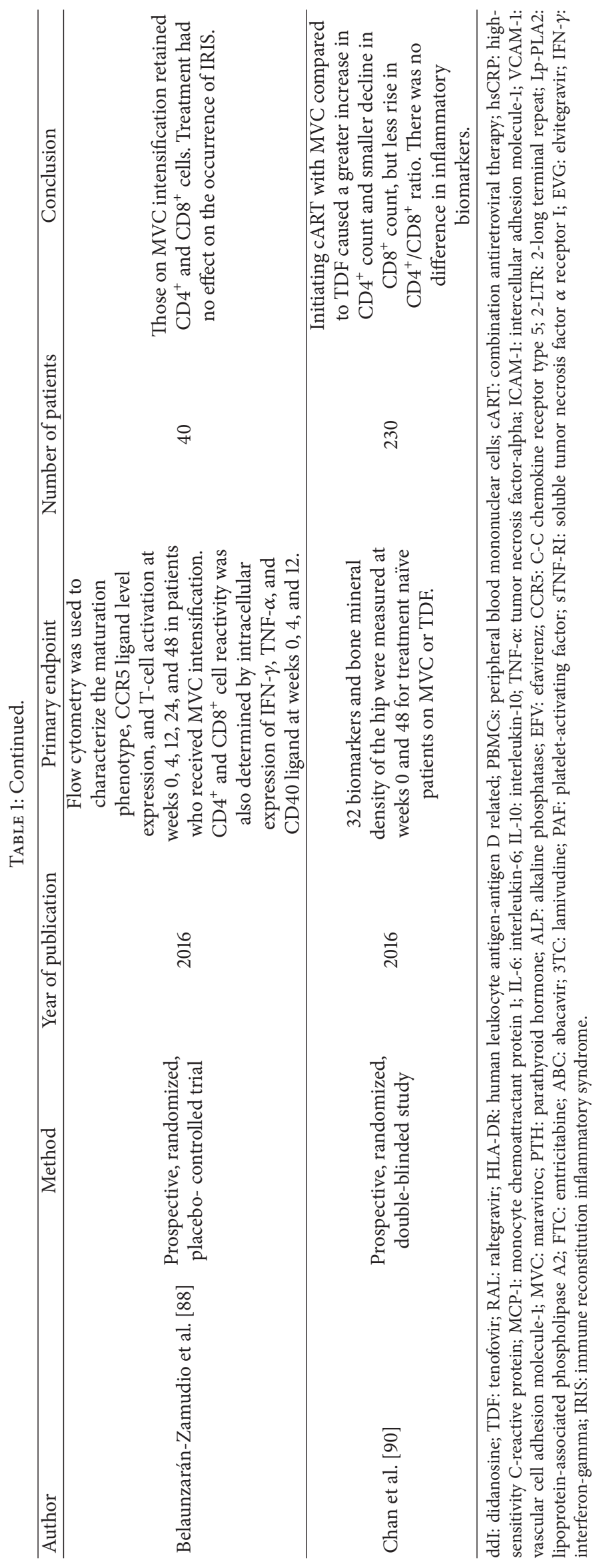


at non-cART intervention was a 12-week, single-arm, openlabel study, whereby Sereti et al. tested the efficacy of IL7 adjunctive therapy on T-cell reconstitution in peripheral blood and gut mucosa in 23 cART suppressed HIV-infected patients with incomplete $\mathrm{CD} 4^{+}$recovery [99]. They observed that administration of r-hIL-7 improved the gut mucosal abnormalities of chronic HIV infection and attenuated the systemic inflammatory and coagulation abnormalities associated with the said gut disease [99].

In the DHHS guidelines, the DHHS does not advocate for cART intensification because this method has not shown consistency in reducing the immune activation nor in increasing T-cell recovery [46]. Furthermore, the guidelines do not recommend switching cART regimens in virally suppressed patients due to the lack of substantial evidence of its effects on the chronic inflammatory response [46]. Also, no instructions for the trending of immune markers in those with chronically suppressed HIV infection have been incorporated into the guidelines because there has yet to be any proven predictability value in morbidity and mortality from doing so [46]. Because of this, the DHHS explains that clinicians should aim at monitoring modifiable risk factors for comorbid conditions [46]. Also, it is recommended that patients with poor $\mathrm{CD}^{+}$recovery should be worked-up for a modifiable cause, most notably adverse events from medications [46].

\section{Conclusion}

Overall, the harmful effects of chronically suppressed HIV infection on inflammation and immune activation must always be considered when managing PLWH. As the population of HIV-infected patients ages, the long-term effects of immune dysregulation will augment the severity of other non-AIDS-related comorbidities. Furthermore, the potential toxic events from certain cART could also contribute towards increased morbidity and mortality compared to the general population in the long run. Although knowledge about this subject is increasing, real life data to suggest appropriate ways to manage this inflammation related to HIV is seriously lacking. There are not enough studies done to establish a consensus to which antiretroviral drug class is best at suppressing the inflammatory response. This is due to the difficulty in finding the right means to accurately measure the chronic inflammatory state. Clinicians will most likely have to settle for a battery of tests that will be validated from collectively trending them over time. At this point, a practical approach to the management of PLWH to best preserve the immune system and control the chronic inflammatory process should be to (1) initiate cART as early as possible, (2) both prevent and treat coinfection, (3) aggressively treat any comorbid condition, (4) advise patients to stop smoking and increase physical activity, and (5) switch cART to the least toxic regimen. It is absolutely essential to conduct more clinical trials to determine which regimens are both most effective and safest at controlling the inflammatory response.

\section{Competing Interests}

The authors declare that they have no competing interests.

\section{References}

[1] Centers for Disease Control and Prevention Morbidity and Mortality Weekly Report, "HIV and AIDS-United States, 1981-2000," http://www.cdc.gov/mmwr/preview/mmwrhtml/ mm5021a2.htm.

[2] H. Samji, A. Cescon, R. S. Hogg et al., "Closing the gap: increases in life expectancy among treated HIV-positive individuals in the United States and Canada," PLoS ONE, vol. 8, no. 12, Article ID e81355, 2013.

[3] P. W. Hunt, J. N. Martin, E. Sinclair et al., "T cell activation is associated with lower $\mathrm{CD} 4^{+} \mathrm{T}$ cell gains in human immunodeficiency virus-infected patients with sustained viral suppression during antiretroviral therapy," The Journal of Infectious Diseases, vol. 187, no. 10, pp. 1534-1543, 2003.

[4] N. I. Wada, L. P. Jacobson, J. B. Margolick et al., "The effect of HAART-induced HIV suppression on circulating markers of inflammation and immune activation," AIDS, vol. 29, no. 4, pp. 463-471, 2015.

[5] C. Tincati, E. Merlini, P. Braidotti et al., "Impaired gut junctional complexes feature late-treated individuals with suboptimal $\mathrm{CD} 4^{+} \mathrm{T}$-cell recovery upon virologically suppressive combination antiretroviral therapy," AIDS, vol. 30, no. 7, pp. 991-1003, 2016.

[6] O. Zaegel-Faucher, S. Bregigeon, C. E. Cano et al., "Impact of hepatitis $\mathrm{C}$ virus coinfection on T-cell dynamics in longterm HIV-suppressors under combined antiretroviral therapy," AIDS, vol. 29, no. 12, pp. 1505-1510, 2015.

[7] G. Marchetti, C. Tincati, and G. Silvestri, "Microbial translocation in the pathogenesis of HIV infection and AIDS," Clinical Microbiology Reviews, vol. 26, no. 1, pp. 2-18, 2013.

[8] A. R. Stacey, P. J. Norris, L. Qin et al., "Induction of a striking systemic cytokine cascade prior to peak viremia in acute human immunodeficiency virus type 1 infection, in contrast to more modest and delayed responses in acute hepatitis B and $\mathrm{C}$ virus infections," Journal of Virology, vol. 83, no. 8, pp. 3719-3733, 2009.

[9] J. M. Brenchley and D. C. Douek, "HIV infection and the gastrointestinal immune system," Mucosal Immunology, vol. 1, no. 1, pp. 23-30, 2008.

[10] E. J. Ciccone, S. W. Read, P. J. Mannon et al., "Cycling of gut mucosal $\mathrm{CD}^{+} \mathrm{T}$ cells decreases after prolonged anti-retroviral therapy and is associated with plasma LPS levels," Mucosal Immunology, vol. 3, no. 2, pp. 172-181, 2010.

[11] N. R. Klatt, N. T. Funderburg, and J. M. Brenchley, "Microbial translocation, immune activation, and HIV disease," Trends in Microbiology, vol. 21, no. 1, pp. 6-13, 2013.

[12] N. G. Sandler, H. Wand, A. Roque et al., "Plasma levels of soluble CD14 independently predict mortality in HIV infection," Journal of Infectious Diseases, vol. 203, no. 6, pp. 780-790, 2011.

[13] P. W. Hunt, E. Sinclair, B. Rodriguez et al., "Gut epithelial barrier dysfunction and innate immune activation predict mortality in treated HIV infection," The Journal of Infectious Diseases, vol. 210, no. 8, pp. 1228-1238, 2014.

[14] M. M. Lederman, N. T. Funderburg, R. P. Sekaly, N. R. Klatt, and P. W. Hunt, "Residual immune dysregulation syndrome in treated HIV infection," Advances in Immunology, vol. 119, pp. 51-83, 2013.

[15] P. W. Hunt, J. N. Martin, E. Sinclair et al., "T cell activation is associated with lower $\mathrm{CD} 4^{+} \mathrm{T}$ cell gains in human immunodeficiency vires-infected patients with sustained viral suppression 
during antiretroviral therapy," Journal of Infectious Diseases, vol. 187, no. 10, pp. 1534-1543, 2003.

[16] M. Masiá, C. Robledano, V. O. de la Tabla et al., "Coinfection with human herpesvirus 8 is associated with persistent inflammation and immune activation in virologically suppressed HIVinfected patients," PLoS ONE, vol. 9, no. 8, Article ID e105442, 2014.

[17] K. Modjarrad and S. H. Vermund, "Effect of treating coinfections on HIV-1 viral load: a systematic review," The Lancet Infectious Diseases, vol. 10, no. 7, pp. 455-463, 2010.

[18] J. R. Deayton, C. A. Sabin, M. A. Johnson, V. C. Emery, P. Wilson, and P. D. Griffiths, "Importance of cytomegalovirus viraemia in risk of disease progression and death in HIVinfected patients receiving highly active antiretroviral therapy," The Lancet, vol. 363, no. 9427, pp. 2116-2121, 2004.

[19] L. Barrett, K. R. Fowke, and M. D. Grant, "Cytomegalovirus, aging, and HIV: a perfect storm," AIDS Reviews, vol. 14, no. 3, pp. 159-167, 2012.

[20] L. H. Kuller, R. Tracy, W. Belloso et al., "Inflammatory and coagulation bio-markers and mortality in patients with HIV infection," PLoS Medicine, vol. 5, no. 10, article e203, 2008.

[21] D. A. Duprez, J. Neuhaus, L. H. Kuller et al., "Inflammation, coagulation and cardiovascular disease in HIV-infected individuals," PLoS ONE, vol. 7, no. 9, Article ID e44454, 2012.

[22] A. R. Tenorio, Y. Zheng, R. J. Bosch et al., "Soluble markers of inflammation and coagulation but not T-cell activation predict non-AIDS-defining morbid events during suppressive antiretroviral treatment," Journal of Infectious Diseases, vol. 210, no. 8, pp. 1248-1259, 2014.

[23] L. Ryom, R. Weber, P. Morlat et al., "Trends in underlying causes of death in people with HIV from 1999 to 2011 (D:A:D): a multicohort collaboration," The Lancet, vol. 384, no. 9939, pp. 241-248, 1999.

[24] P. Morlat, C. Roussillon, S. Henard et al., "Causes of death among HIV-infected patients in France in 2010 (national survey): trends since 2000," AIDS, vol. 28, no. 8, pp. 1181-1191, 2014.

[25] B. Hasse, B. Ledergerber, H. Furrer et al., "Morbidity and aging in HIV-infected persons: the swiss HIV cohort study," Clinical Infectious Diseases, vol. 53, no. 11, pp. 1130-1139, 2011.

[26] G. Guaraldi, G. Orlando, S. Zona et al., "Premature age-related comorbidities among HIV-infected persons compared with the general population," Clinical Infectious Diseases, vol. 53, no. 11, pp. 1120-1126, 2011.

[27] M. S. Freiberg, C. C. Chang, L. H. Kuller et al., "HIV infection and the risk of acute myocardial infarction," JAMA Internal Medicine, vol. 173, no. 8, pp. 614-622, 2013.

[28] V. A. Triant, H. Lee, C. Hadigan, and S. K. Grinspoon, "Increased acute myocardial infarction rates and cardiovascular risk factors among patients with human immunodeficiency virus disease," Journal of Clinical Endocrinology and Metabolism, vol. 92, no. 7, pp. 2506-2512, 2007.

[29] N. L. Okeke, C. B. Hicks, M. S. McKellar, V. G. Fowler Jr., and J. J. Federspiel, "History of AIDS in HIV-infected patients is associated with higher in-hospital mortality following admission for acute myocardial infarction and stroke," Journal of Infectious Diseases, vol. 213, no. 12, pp. 1955-1961, 2016.

[30] M. S. Shiels, R. M. Pfeiffer, M. H. Gail et al., "Cancer burden in the HIV-infected population in the United States," JNCI Journal of the National Cancer Institute, vol. 103, no. 9, pp. 753-762, 2011.
[31] A. J. Rodger, R. Lodwick, M. Schechter et al., "Mortality in well controlled HIV in the continuous antiretroviral therapy arms of the SMART and ESPRIT trials compared with the general population," AIDS, vol. 27, no. 6, pp. 973-979, 2013.

[32] R. Dubrow, M. J. Silverberg, L. S. Park, K. Crothers, and A. C. Justice, "HIV infection, aging, and immune function: implications for cancer risk and prevention," Current Opinion in Oncology, vol. 24, no. 5, pp. 506-516, 2012.

[33] Z. Tyerman and D. M. Aboulafia, "Review of screening guidelines for non-AIDS-defining malignancies: evoking issues in the era of highly active antiretroviral therapy," AIDS Reviews, vol.14, no. 1, pp. 3-16, 2012.

[34] T. Powles, D. Robinson, J. Stebbing et al., "Highly active antiretroviral therapy and the incidence of non-AIDS-defining cancers in people with HIV infection," Journal of Clinical Oncology, vol. 27, no. 6, pp. 884-890, 2009.

[35] L. Shepherd, Á. Borges, B. Ledergerber et al., "Infection-related and -unrelated malignancies, HIV and the aging population," HIV Medications, vol. 17, no. 8, pp. 590-600, 2016.

[36] D. J. Riedel, L. S. Tang, and A. F. Rositch, "The role of viral coinfection in HIV-associated non-AIDS-related cancers," Current HIV/AIDS Reports, vol. 12, no. 3, pp. 362-372, 2015.

[37] A. Carbone, A. Gloghini, and G. Dotti, "EBV-associated lymphoproliferative disorders: classification and treatment," Oncologist, vol. 13, no. 5, pp. 577-585, 2008.

[38] C. Piketty, H. Selinger-Leneman, A.-M. Bouvier et al., "Incidence of HIV-related anal cancer remains increased despite long-term combined antiretroviral treatment: results from the french hospital database on HIV," Journal of Clinical Oncology, vol. 30, no. 35, pp. 4360-4366, 2012.

[39] B. Hasse, B. Ledergerber, H. Furrer et al., "Morbidity and aging in HIV-infected persons: the Swiss HIV cohort study," Clinical Infectious Diseases, vol. 53, no. 11, pp. 1130-1139, 2011.

[40] A. D. Gutierrez and A. Balasubramanyam, "Dysregulation of glucose metabolism in HIV patients: epidemiology, mechanisms, and management," Endocrine, vol. 41, no. 1, pp. 1-10, 2012.

[41] M. Giralt, P. Domingo, and F. Villarroya, "Adipose tissue biology and HIV-infection," Best Practice \& Research Clinical Endocrinology \& Metabolism, vol. 25, no. 3, pp. 487-499, 2011.

[42] W. J. Towner, L. Xu, W. A. Leyden et al., "The effect of HIV infection, immunodeficiency, and antiretroviral therapy on the risk of hepatic dysfunction," Journal of Acquired Immune Deficiency Syndromes, vol. 60, no. 3, pp. 321-327, 2012.

[43] G. Marchetti, A. Cozzi-Lepri, C. Tincati et al., "Immune activation and microbial translocation in liver disease progression in HIV/hepatitis co-infected patients: results from the Icona Foundation study," BMC Infectious Diseases, vol. 14, article 79, 2014.

[44] J. P. Anderson, C. R. Horsburgh Jr., P. L. Williams et al., "CD4 recovery on antiretroviral therapy is associated with decreased progression to liver disease among hepatitis $\mathrm{C}$ virus-infected injecting drug users," Open Forum Infectious Diseases, vol. 2, no. 1, p. ofv019, 2015.

[45] C. F. Kelley, C. M. R. Kitchen, P. W. Hunt et al., "Incomplete peripheral $\mathrm{CD} 4^{+}$cell count restoration in HIV-infected patients receiving long-term antiretroviral treatment," Clinical Infectious Diseases, vol. 48, no. 6, pp. 787-794, 2009.

[46] Panel on Antiretroviral Guidelines for Adults and Adolescents, "Guidelines for the use of antiretroviral agents in HIV-1infected adults and adolescents," Department of Health and Human Services, http://www.aidsinfo.nih.gov/ContentFiles/ AdultandAdolescentGL.pdf. 
[47] C. A. Sabin, C. J. Smith, A. d'Arminio Monforte et al., "Response to combination antiretroviral therapy: variation by age," AIDS, vol. 22, no. 12, pp. 1463-1473, 2008.

[48] K. N. Althoff, A. C. Justice, S. J. Gange et al., "Virologic and immunologic response to HAART, by age and regimen class," AIDS, vol. 24, no. 16, pp. 2469-2479, 2010.

[49] J. D. Lundgren, A. G. Babiker, F. Gordin et al., "Initiation of antiretroviral therapy in early asymptomatic HIV infection," The New England Journal of Medicine, vol. 373, no. 9, pp. 795807, 2015.

[50] TEMPRANO ANRS Study Group, C. Danel, R. Moh et al., "A trial of early antiretrovirals and isoniazid preventive therapy in Africa," The New England Journal of Medicine, vol. 373, no. 9, pp. 808-822, 2015.

[51] S. G. Deeks, "HIV infection, inflammation, immunosenescence, and aging," Annual Review of Medicine, vol. 62, no. 1, pp. 141-155, 2011.

[52] N. Friis-Møller, R. Weber, P. Reiss et al., "Cardiovascular disease risk factors in HIV patients-association with antiretroviral therapy. Results from the DAD study," AIDS, vol. 17, no. 8, pp. 1179-1193, 2003.

[53] S. W. Worm, C. Sabin, R. Weber et al., "Risk of myocardial infarction in patients with HIV infection exposed to specific individual antiretroviral drugs from the 3 major drug classes: the data collection on adverse events of anti-HIV drugs (D:A:D) Study," Journal of Infectious Diseases, vol. 201, no. 3, pp. 318-330, 2010.

[54] S. Lang, M. Mary-Krause, L. Cotte et al., "Impact of individual antiretroviral drugs on the risk of myocardial infarction in human immunodeficiency virus-infected patients: A casecontrol study nested within the French hospital database on HIV ANRS cohort $\mathrm{CO}_{4}$," Archives of Internal Medicine, vol. 170, no. 14, pp. 1228-1238, 2010.

[55] M. Durand, O. Sheehy, J.-G. Baril, J. Lelorier, and C. L. Tremblay, "Association between HIV infection, antiretroviral therapy, and risk of acute myocardial infarction: a cohort and nested casecontrol study using Québec's Public Health Insurance database," Journal of Acquired Immune Deficiency Syndromes, vol. 57, no. 3, pp. 245-253, 2011.

[56] J. L. Marcus, R. S. Neugebauer, W. A. Leyden et al., "Use of abacavir and risk of cardiovascular disease among HIV-infected individuals," Journal of Acquired Immune Deficiency Syndromes, vol. 71, no. 4, pp. 413-419, 2016.

[57] The SMART/INSIGHT and the D:A:D Study Groups; TSI at DADSG, "Use of nucleoside reverse transcriptase inhibitors and risk of myocardial infarction in HIV-infected patients," AIDS, vol. 22, no. 14, pp. F17-F24, 2008.

[58] N. Obel, D. K. Farkas, G. Kronborg et al., "Abacavir and risk of myocardial infarction in HIV-infected patients on highly active antiretroviral therapy: a population-based nationwide cohort study," HIV Medicine, vol. 11, no. 2, pp. 130-136, 2010.

[59] A. I. Choi, E. Vittinghoff, S. G. Deeks, C. C. Weekley, Y. Li, and M. G. Shlipak, "Cardiovascular risks associated with abacavir and tenofovir exposure in HIV-infected persons," AIDS, vol. 25, no. 10, pp. 1289-1298, 2011.

[60] D. Costagliola, S. Lang, M. Mary-Krause, and F. Boccara, "Abacavir and cardiovascular risk: reviewing the evidence," Current HIV/AIDS Reports, vol. 7, no. 3, pp. 127-133, 2010.

[61] C. H. Brothers, J. E. Hernandez, A. G. Cutrell et al., "Risk of myocardial infarction and abacavir therapy: no increased risk across 52 glaxosmithkline-sponsored clinical trials in adult subjects," Journal of Acquired Immune Deficiency Syndromes, vol. 51, no. 1, pp. 20-28, 2009.

[62] R. J. Bedimo, A. O. Westfall, H. Drechsler, G. Vidiella, and P. Tebas, "Abacavir use and risk of acute myocardial infarction and cerebrovascular events in the highly active antiretroviral therapy era," Clinical Infectious Diseases, vol. 53, no. 1, pp. 8491, 2011.

[63] H. J. Ribaudo, C. A. Benson, Y. Zheng et al., "No risk of myocardial infarction associated with initial antiretroviral treatment containing abacavir: short and long-term results from ACTG A5001/ALLRT,' Clinical Infectious Diseases, vol. 52, no. 7, pp. 929-940, 2011.

[64] X. Ding, E. Andraca-Carrera, C. Cooper et al., "No association of abacavir use with myocardial infarction: findings of an FDA meta-analysis," Journal of Acquired Immune Deficiency Syndromes, vol. 61, no. 4, pp. 441-447, 2012.

[65] L. Ryom, J. D. Lundgren, S. De Wit et al., "Use of antiretroviral therapy and risk of end-stage liver disease and hepatocellular carcinoma in HIV-positive persons," AIDS, vol. 30, no. 11, pp. 1731-1743, 2016.

[66] S. De Wit, C. Sabin, R. Weber et al., "Incidence and risk factors for new-onset diabetes in HIV-infected patients: the Data Collection on Adverse Events of Anti-HIV Drugs (D:A:D) study," Diabetes Care, vol. 31, no. 6, pp. 1224-1229, 2008.

[67] F. Gutiérrez and M. Masiá, "The role of HIV and antiretroviral therapy in bone disease," AIDS Reviews, vol. 13, no. 2, pp. 109118, 2011.

[68] T. T. Brown and R. B. Qaqish, "Antiretroviral therapy and the prevalence of osteopenia and osteoporosis: a meta-analytic review," AIDS, vol. 20, no. 17, pp. 2165-2174, 2006.

[69] S. Perrot, E. Aslangul, T. Szwebel, N. Caillat-Vigneron, and C. Le Jeunne, "Bone pain due to fractures revealing osteomalacia related to tenofovir-induced proximal renal tubular dysfunction in a human immunodeficiency virus-infected patient," Journal of Clinical Rheumatology, vol. 15, no. 2, pp. 72-74, 2009.

[70] L. C. Herlitz, S. Mohan, M. B. Stokes, J. Radhakrishnan, V. D. D'Agati, and G. S. Markowitz, "Tenofovir nephrotoxicity: acute tubular necrosis with distinctive clinical, pathological, and mitochondrial abnormalities," Kidney International, vol. 78, no. 11, pp. 1171-1177, 2010.

[71] P. E. Sax, D. Wohl, M. T. Yin et al., “Tenofovir alafenamide versus tenofovir disoproxil fumarate, coformulated with elvitegravir, cobicistat, and emtricitabine, for initial treatment of HIV-1 infection: two randomised, double-blind, phase 3, noninferiority trials," The Lancet, vol. 385, no. 9987, pp. 2606-2615, 2015.

[72] E. Leeansyah, P. U. Cameron, A. Solomon et al., "Inhibition of telomerase activity by HIV nucleos(t)ide reverse transcriptase inhibitors: a potential factor contributing to HIV-associated accelerated aging," Journal of Infectious Diseases, vol. 207, no. 7, pp. 1157-1165, 2013.

[73] J. C. Y. Liu, J. M. Leung, D. A. Ngan et al., "Absolute leukocyte telomere length in HIV-infected and uninfected individuals: evidence of accelerated cell senescence in HIV-associated chronic obstructive pulmonary disease," PLoS ONE, vol. 10, no. 4, Article ID e0124426, 2015.

[74] D. A. L. Zanet, A. Thorne, J. Singer et al., "Association between short leukocyte telomere length and HIV infection in a cohort study: no evidence of a relationship with antiretroviral therapy," Clinical Infectious Diseases, vol. 58, no. 9, pp. 1322-1332, 2014. 
[75] A. Anderson, J. Ludicello, A. Kallianpur et al., "CNS drug distrubtion and CSF inflammation during supressive antriretroviral therapy," in Proceedings of the Conference of Retroviruses and Opportunistic Infections (CROI '16), Abstract no. 412, Boston, Mass, USA, February 2016.

[76] D. Chege, C. Kovacs, C. la Porte et al., "Effect of raltegravir intensification on HIV proviral DNA in the blood and gut mucosa of men on long-term therapy: a randomized controlled trial," AIDS, vol. 26, no. 2, pp. 167-174, 2012.

[77] S. A. Yukl, A. K. Shergill, K. McQuaid et al., "Effect of raltegravir-containing intensification on HIV burden and T-cell activation in multiple gut sites of HIV-positive adults on suppressive antiretroviral therapy," AIDS, vol. 24 , no. 16, pp. 24512460, 2010.

[78] K. B. Patterson, H. A. Prince, T. Stevens et al., "Differential penetration of raltegravir throughout gastrointestinal tissue: implications for eradication and cure," AIDS, vol. 27, no. 9, pp. 1413-1419, 2013.

[79] R. T. Gandhi, L. Zheng, R. J. Bosch et al., "The Effect of Raltegravir Intensification on Low-level Residual Viremia in HIVInfected Patients on Antiretroviral Therapy: A Randomized Controlled Trial," PLoS Medicine, vol. 7, no. 8, p. e1000321, 2010.

[80] H. Hatano, M. C. Strain, R. Scherzer et al., "Increase in 2long terminal repeat circles and decrease in D-dimer after raltegravir intensification in patients with treated HIV infection: a randomized, placebo-controlled trial," Journal of Infectious Diseases, vol. 208, no. 9, pp. 1436-1442, 2013.

[81] H. Hatano, T. L. Hayes, V. Dahl et al., "A randomized, controlled trial of raltegravir intensification in antiretroviral-treated, HIVinfected patients with a suboptimal $\mathrm{CD} 4^{+} \mathrm{T}$ cell response," The Journal of Infectious Diseases, vol. 203, no. 7, pp. 960-968, 2011.

[82] E. Martínez, P. M. D’Albuquerque, J. M. Llibre et al., "Changes in cardiovascular biomarkers in HIV-infected patients switching from ritonavir-boosted protease inhibitors to raltegravir," AIDS, vol. 26, no. 18, pp. 2315-2326, 2012.

[83] E. F. Silva, I. Charreau, B. Gourmel et al., "Decreases in inflammatory and coagulation biomarkers levels in HIV-infected patients switching from enfuvirtide to raltegravir: ANRS 138 substudy," Journal of Infectious Diseases, vol. 208, no. 6, pp. 892897, 2013.

[84] J. E. Lake, G. A. McComsey, T. Hulgan et al., "Switch to raltegravir decreases soluble CD14 in virologically suppressed overweight women: the Women, Integrase and Fat Accumulation Trial," HIV Medicine, vol. 15, no. 7, pp. 431-441, 2014.

[85] S. K. Gupta, D. Mi, S. M. Moe, M. P. Dubé, and Z. Liu, "Effects of switching from efavirenz to raltegravir on endothelial function, bone mineral metabolism, inflammation, and renal function: a randomized, controlled trial," Journal of Acquired Immune Deficiency Syndromes, vol. 64, no. 3, pp. 279-283, 2013.

[86] L. Cuzin, S. Trabelsi, P. Delobel et al., "Maraviroc intensification of stable antiviral therapy in HIV-1-infected patients with poor immune restoration: MARIMUNO-ANRS 145 study," Journal of Acquired Immune Deficiency Syndromes, vol. 61, no. 5, pp. 557564, 2012.

[87] P. W. Hunt, N. S. Shulman, T. L. Hayes et al., "The immunologic effects of maraviroc intensification in treated HIV-infected individuals with incomplete CD41 T-cell recovery: A randomized trial," Blood, vol. 121, no. 23, article no. 4635, 2013.

[88] P. Belaunzarán-Zamudio, L. Azzoni, J. Sierra-Madero et al., "Maraviroc and immune recovery in advanced AIDS," in Proceedings of the Conference of Retroviruses and Opportunistic
Infections (CROI '16), Abstract no. 296, Boston, Mass, USA, February 2016.

[89] C. O. Hileman, B. Kinley, V. Scharen-Guivel et al., "Differential reduction in monocyte activation and vascular inflammation with integrase inhibitor-based initial antiretroviral therapy among HIV-infected individuals," Journal of Infectious Diseases, vol. 212, no. 3, pp. 345-354, 2015.

[90] E. Chan, P. Mirmonsef, T. Brown et al., "Immunologic effects of Maraviroc vs Tenofovir and association with bone loss," in Proceedings of the Conference of Retroviruses and Opportunistic Infections (CROI '16), Abstract no. 695LB, Boston, Mass, USA, February 2016.

[91] V. D. Papakonstantinou, M. Chini, N. Mangafas et al., "In vivo effect of two first-line ART regimens on inflammatory mediators in male HIV patients," Lipids in Health and Disease, vol. 13, article 90, 2014.

[92] A. Barrios, A. Rendón, E. Negredo et al., "Paradoxical CD4 ${ }^{+}$ T-cell decline in HIV-infected patients with complete virus suppression taking tenofovir and didanosine," AIDS, vol. 19, no. 6, pp. 569-575, 2005.

[93] L. Calza, E. Vanino, C. Salvadori et al., "Tenofovir/emtricitabine/efavirenz plus rosuvastatin decrease serum levels of inflammatory markers more than antiretroviral drugs alone in antiretroviral therapy-naive hiv-infected patients," HIV Clinical Trials, vol. 15, no. 1, pp. 1-13, 2014.

[94] A. R. Eckard, Y. Jiang, S. M. Debanne, N. T. Funderburg, and G. A. Mccomsey, "Effect of 24 weeks of statin therapy on systemic and vascular inflammation in HIV-infected subjects receiving antiretroviral therapy," Journal of Infectious Diseases, vol. 209, no. 8, pp. 1156-1164, 2014.

[95] N. T. Funderburg, Y. Jiang, S. M. Debanne et al., "Rosuvastatin treatment reduces markers of monocyte activation in HIVinfected subjects on antiretroviral therapy," Clinical Infectious Diseases, vol. 58, no. 4, pp. 588-595, 2014.

[96] J. S. Wooten, P. Nambi, B. K. Gillard et al., "Intensive lifestyle modification reduces Lp-PLA2 in dyslipidemic HIV/HAART patients," Medicine \& Science in Sports \& Exercise, vol. 45, no. 6, pp. 1043-1050, 2013.

[97] R. Valiathan, M. J. Miguez, B. Patel, K. L. Arheart, and D. Asthana, "Tobacco smoking increases immune activation and impairs T-cell function in HIV infected patients on antiretrovirals: a cross-sectional pilot study," PLoS ONE, vol. 9, no. 5, Article ID e97698, 2014.

[98] J. Villar-García, J. J. Hernández, R. Güerri-Fernández et al., "Effect of probiotics (Saccharomyces boulardii) on microbial translocation and inflammation in HIV-treated patients: a double-blind, randomized, placebo-controlled trial," JAIDS Journal of Acquired Immune Deficiency Syndromes, vol. 68, no. 3, pp. 256-263, 2015.

[99] I. Sereti, J. D. Estes, W. L. Thompson et al., "Decreases in colonic and systemic inflammation in chronic HIV infection after IL7 administration," PLoS Pathogens, vol. 10, no. 1, Article ID e1003890, 2014. 

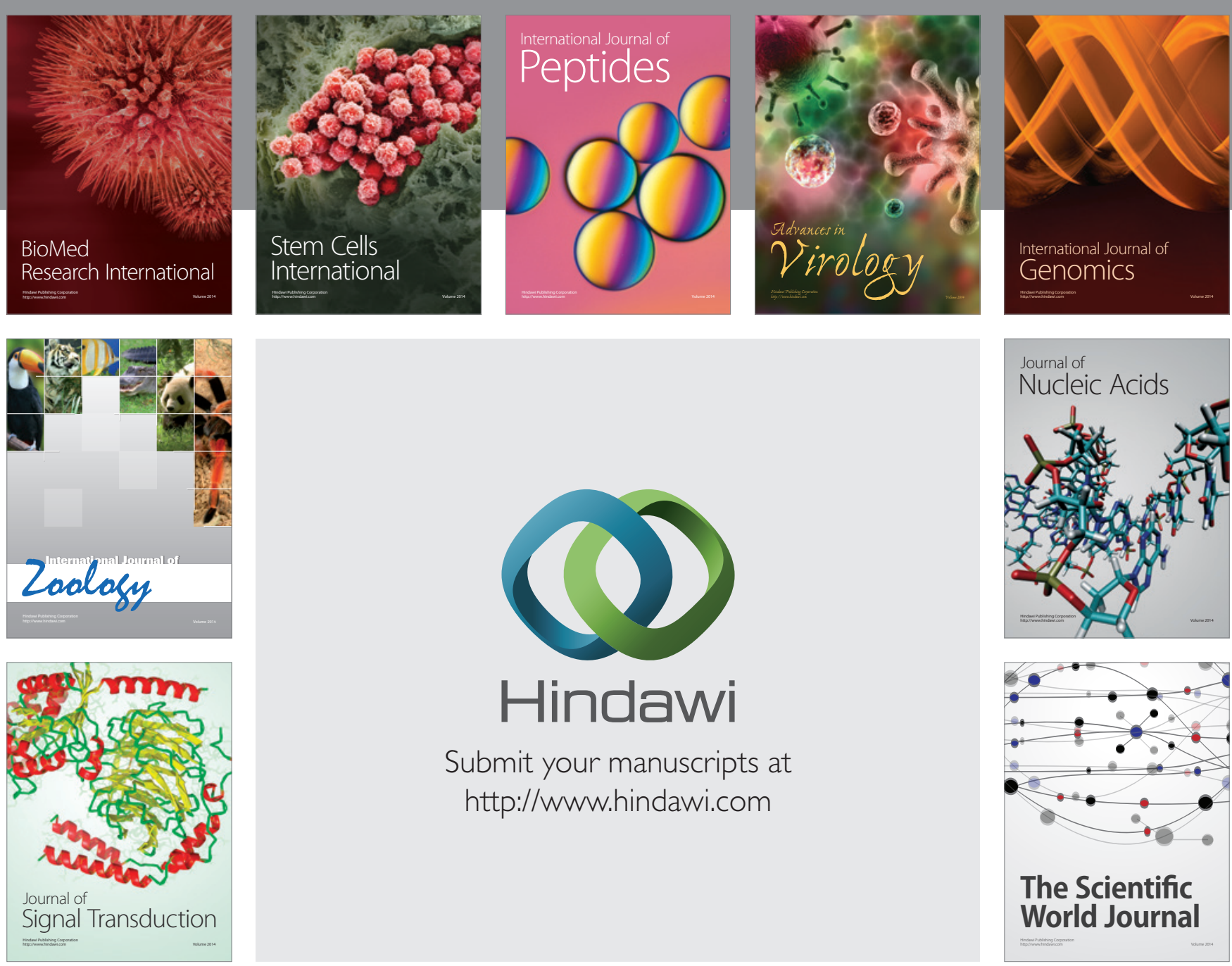

Submit your manuscripts at

http://www.hindawi.com
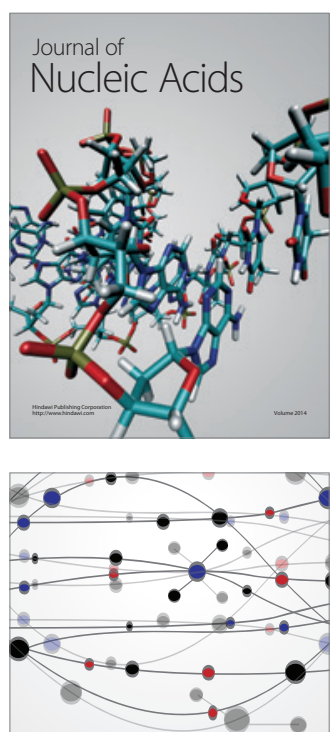

The Scientific World Journal
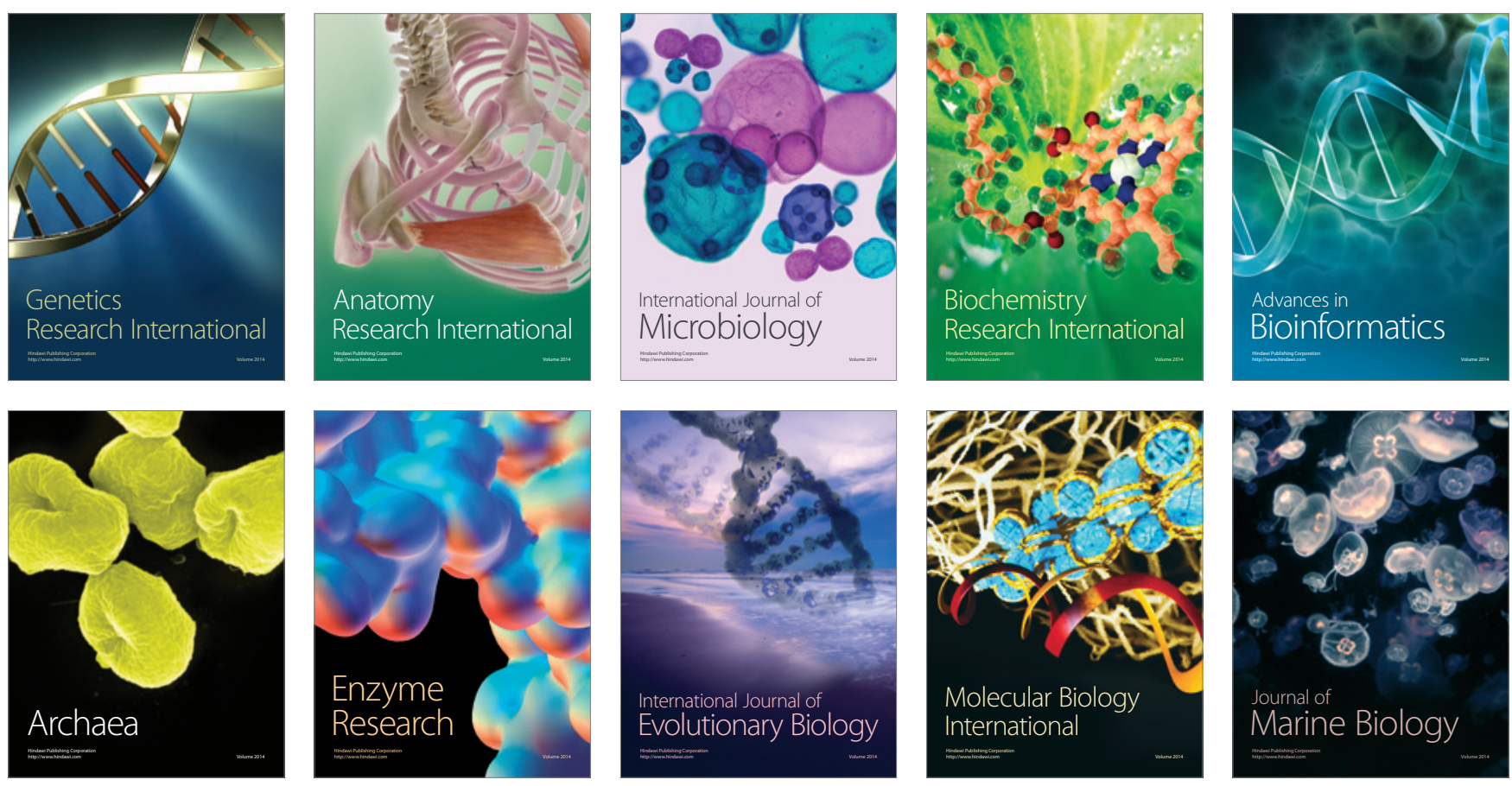